\title{
SEALING ABILITY OF MINERAL TRIOXIDE AGGREGATE, CALCIUM PHOSPHATE CEMENT, AND GLASS IONOMER CEMENT IN THE REPAIR OF FURCATION PERFORATIONS
}

\author{
Prabath Singh', Joseph Paul ${ }^{2}$, Abdul Aziz Al- Khuraif ${ }^{3}$, Sajith Vellappally,3, Hassan Suliman Halawany ${ }^{5,6}$, \\ Mohamed Hashim $^{3,4}$, Nimmi Biju Abraham ${ }^{5}$, Vimal Jacob ${ }^{5}$, Rooban Thavarajah ${ }^{7}$
}

\begin{abstract}
Department of Conservative Dentistry and Endodontics, Amrita School of Dentistry, Amrita Institute of Medical Sciences and Research Center, Cochin, Kerala, India ${ }^{1}$; Department of Conservative Dentistry and Endodontics, VMS Dental College, Salem, Tamil Nadu, India²; Dental Biomaterials Research Chair, College of Applied Medical Sciences, King Saud University, Riyadh, Saudi Arabia3; Department of Dental Health, College of Applied Medical Sciences, King Saud University, Riyadh, Saudi Arabia ${ }^{4}$; Dental Caries Research Chair, College of Dentistry, King Saud University, Riyadh, Saudi Arabia ${ }^{5}$; Department of Periodontics and Community Dentistry, College of Dentistry, King Saud University, Riyadh, Saudi Arabia ${ }^{6}$; Department of Oral and Maxillofacial Pathology, Marundeeshwara Oral Pathology Services and Analytics, Chennai, Tamil Nadu, India $^{7}$
\end{abstract}

Summary: Objectives: The purpose of this study was to evaluate the in vitro sealing ability of three repair materials. Mineral trioxide aggregate (MTA; Group A), calcium phosphate cement (CPC; Group B), and light cured glass ionomer cement (GIC; Group C) when used to repair the perforation created in the pulpal floor of fifty extracted human permanent molars. Materials and methods: Preparation of access openings and furcation perforations were done, and the teeth divided into five experimental groups (A, B, C) including two controls (D, E) with ten samples in each group randomly. Following the repair procedure, the pulp chambers and access openings were filled with composite resin and immersed in $2 \%$ methylene blue solution for 48 hours. The teeth were sectioned longitudinally and the linear dye penetration measured under a stereomicroscope. Results: The comparison of the linear length of micro-leakage $(\mathrm{mm})$ among the experimental groups revealed no significant difference $(p=0.332)$. On calculating the percentage of depth of leakage to the total length of the perforation, it was observed that the mean leakage was 35.5\% in Group A, 53.6\% in Group B and the highest, 87.5\% in Group C. The mean of leakage percentage was statistically significant by Kruskal-Wallis test $(\mathrm{p}=0.003)$. The results indicated that the dye penetration used as furcation perforation repair material was least with mineral trioxide aggregate. Comparing the depth of penetration of dye, $50 \%$ of the Group A samples showed less than $25 \%$ of depth penetration. While $40 \%$ of Group $\mathrm{B}$ cases had more than $50 \%$ dye penetration. In our study, all Group $\mathrm{C}$ teeth had $\geq 50 \%$ dye penetration. Conclusions: The present study indicated that GIC had the greatest dye penetration followed by CPC and MTA. Mineral trioxide aggregate and calcium phosphate cement had comparatively better sealing ability than glass ionomer cement.

Keywords: Furcation defects; Mineral trioxide aggregate; Calcium phosphate cement; Glass ionomer cement; In vitro; Iatrogenic

\section{Introduction}

Furcation perforations constitute a procedural problem that may arise iatrogenically during endodontic therapy (1). It has been reported as the second leading cause of endodontic failures following obturation and this constitute up to $9.6 \%$ of all unsuccessful cases (2). Such perforations occur as an artificial communication between the pulpal space and periodontal ligament space through the floor of the pulp chamber. A cascade of complications may arise as sequelae to a perforation which includes chronic inflammation, damage to the periodontal attachment, proliferation of the epithelial tissue, and continuous osseous break down with eventual loss of teeth (3). Appropriate and immediate treatment are necessary in order to retain the involved tooth and for favorable prognosis. Various surgical and nonsurgical methods have been evaluated for the repair of furcation perforations. Non-surgical approaches by the placement of repair materials at perforation sites are preferred in cases where there is lack of surgical accessibility (4). While surgical approach, if accessible, leads to chronic pocket formation (5), nonsurgical repair faces with the difficulty of extrusion of filling material into the periodontal space which hinders periodontal reattachment (1). 
The prognosis of endodontically treated tooth with a perforation depends on several factors such as time of occurrence, size, shape and location of the perforation (4). In addition, the choice of sealing material is also a crucial factor that influences the outcome of treatment (6). Ideally, an effective repair material should provide an adequate seal with less setting time, be biocompatible, nontoxic, noncarcinogenic, relatively inexpensive, bacteriostatic, easy to procure, simple to handle, and possess the ability to induce osteogenesis and cementogenesis. It should also serve as an effective barrier or matrix between root canal filling and the tissues (7). No material was found to possess all the above mentioned properties. In search for the ideal requirements, a plethora of sealing materials and techniques has been tested for the restoration of the perforations over the years with varying degrees of success (4).

Mineral trioxide aggregate (MTA) has been recommended as a novel repair material since it was introduced for the repair of root perforations in 1993 at Loma Linda University (8). The repair of furcal perforations with MTA was first investigated in 1995 by Pitt Ford et al. (9). Its good biocompatibility has been demonstrated by its ability to induce the formation of hydroxyapatite when exposed to synthetic tissue fluids. A successful treatment with this water based cement depends on repairing the perforation site before contamination occurs (10).

Calcium phosphate cements (CPC) were first reported by Brown and Chow in 1986 as a self-setting, bioactive and biodegradable grafting material which consists of an equimolar mixture of tricalcium phosphate and dicalcium phosphate (anhydrous or dihydrate). It is available in the form of powder and liquid, which when mixed sets primarily as hydroxyapatite. This mouldable treatment modality is reported to possess excellent biological properties and can potentially be replaced by bone via osteoconduction and concurrent cement absorption after a period of time (11). The use of an experimental CPC and MTA for furcation perforation repair revealed no significant differences in terms of bone reorganization (12).

Light cured glass ionomer cement (GIC) is considered as a hybrid cement which became commercially available in the late 1980s. It consists of a complex mixture of components such as polyacrylic acid, photocurable monomer (hydroxyethyl methacrylate; HEMA), ion leachable glass and water (13). A study done by Alhadainy and Himel (14) found that light cured GIC exhibited the least dye penetration and provides better sealing ability when compared to amalgam or cavit and showed superiority over chemically cured GIC (15) following an in vitro repair of furcation perforations.

The purpose of the present study was to evaluate the in vitro sealing ability of MTA, CPC and light cured GIC when used to repair the perforation created in the pulpal floor of extracted human permanent molars in a simulated clinical situation. The null hypothesis tested was that there would be no difference in the sealing ability of the materials.

\section{Materials and methods}

\section{Tooth preparation}

This study was approved by the ethical committee of Amrita School of Dentistry, Cochin, India and was conducted in the Department of Conservative Dentistry and Endodontics. Fifty, conveniently sampled, human maxillary and mandibular molars that were scheduled for extraction were used for this study. The intact teeth were atraumatically extracted and stored in $10 \%$ formalin for a minimum of seven days (16). Calculus and soft tissue tags were removed by ultrasonic scaling. The teeth were washed with tap water to elude the formalin fixative and were stored in normal saline until further use. Collection, disinfection, storage and handling of extracted teeth for the study were followed according to the guidelines recommended by Kohli et al. (17).

In all collected teeth for the experiment, standard coronal access openings were prepared to use a size \#557 straight fissure carbide bur (SS White Burs, Inc. Lakewood, NJ, USA) and perforations were made directly in the center of the pulp chamber floor using \#2 round bur (carbide burs FG-2; SS white burs, Inc. Lakewood, NJ) in a high speed hand piece with water coolant. The widths of all perforations were standardized to the diameter of \#2 round burs. The depth of the perforation varied with the dentin-cementum thickness in the furcation area. The area was then dried with compressed air.

\section{Repair procedures}

The teeth were randomly divided into 5 experimental groups with 10 samples in each group as: Group A (defect repaired with MTA), Group B (defect repaired with CPC), Group C (defect repaired with GIC), Group D (positive controls) and Group E (negative controls). The distribution of maxillary and mandibular teeth was alike for each group. The manufacturer's instructions were followed while dispensing, preparing, mixing and placing the materials.

Group A: MTA (ProRoot, Dentsply, Switzerland) powder was mixed on a paper pad with distilled water in a $3: 1$ powder water ratio. When the mixture exhibited putty like consistency after about $30 \mathrm{~s}$ mixing time, it was immediately placed into the perforation with a carrier gun. Endodontic pluggers followed by a wet cotton pellet were used to condense the material gently into the perforation site. A moistened cotton pellet was placed in the pulp chamber.

Group B: Fully injectable CPC was obtained from Sree Chitra Tirunal Institute of Medical Science, Trivandrum, India and this formulation was delivered to the defect site through an 18 gauge needle from a $3 \mathrm{ml}$ disposable syringe and then allowed to set for about 20 minutes. Additional CPC was injected until the perforation was entirely filled.

Group C: Light cured GIC (Vitrebond 3M, St Paul, MN) was used to repair the perforation defect in this group. It 
was prepared by mixing a scoop of powder in two drops of liquid. A small amount of material was carried into the pulp chamber on the tip of a straight probe and allowed to flow apically in the perforation. The GIC was then light cured for $30 \mathrm{~s}$ with a compact visible light-curing unit (Ivoclar Vivadent, Schaan, Liechtenstein). Incremental layers were applied, and light cured until the perforation was filled.

Care was taken to fill all experimental perforations using the repair materials (group A, B, C) to the level of pulpal floor. The root was moistened with cotton pellet inertly to simulate the clinical situation during the repair process. No effort was made to prevent the overextension of the repair material. After completion of the repair, pulp chamber and access preparation of all teeth were filled with light cured composite resin (3M ESPE, St Paul, MN).

Group D: Perforated teeth received no furcation repair or a filling and served as a positive control to demonstrate that dye could flow into the perforations. A cotton pellet was placed in the pulp chamber to cover the perforation. A thin layer of calcium hydroxide liner (Dycal, Dentsply Caulk Ltd, Milford, DE) followed to prevent the composite resin from occluding the defect. The pulp chamber and access preparation of all teeth was filled with light cured composite resin (3M ESPE, St Paul, MN).

Group $\boldsymbol{E}$ : For the negative control group E, the unprepared teeth were fully painted with two layers of colored nail varnish (Starry pink, Revlon color cosmetics, NY, USA). This ensured an adequate barrier against dye penetration. No further treatment was done.

\section{Evaluation of experimental groups}

To avoid dye penetration through other areas, all experimental teeth were then coated homogeneously with two coats of nail varnish except for 1 to $2 \mathrm{~mm}$ around the perforations. After $24 \mathrm{~h}$ of drying, all teeth were immersed in a $2 \%$ methylene blue solution (Sigma- Aldrich (M), Sdn Bhd, Malaysia) for $48 \mathrm{~h}$. Prior to dyeing, drying for $24 \mathrm{hrs}$ was done to ensure that dye did not spread to other areas by capillary action or dispersed in to the area of interest by any other means. Moreover this ensured complete curing of the varnish (barrier). After removal from the dye, the teeth were rinsed with water, dried at room temperature for 24 hours and rooted individually in clear orthodontic resin (Dentsply Caulk Ltd, Milford, DE). After curing, the resin blocks were longitudinally sectioned all the way through the repaired perforations in a bucco-lingual direction; parallel to the long axis of the tooth using a precision diamond saw (Isomet 2000, Buehler Ltd., Lake Bluff, IL, USA) under copious water cooling.

The sections revealed the filling material between the two axial walls of the perforation. One end of the perforation was towards the pulp chamber (coronal) and the other end towards the furcation (apical). The sections were examined under optical stereomicroscope (Zeiss Axioskop, Munich, Germany) at $40 \times( \pm 10)$ magnification. The perforation wall that exhibited the greatest penetration of the dye was chosen for evaluation.

The length of the perforation wall and the amount of linear dye penetration along the perforation walls from the
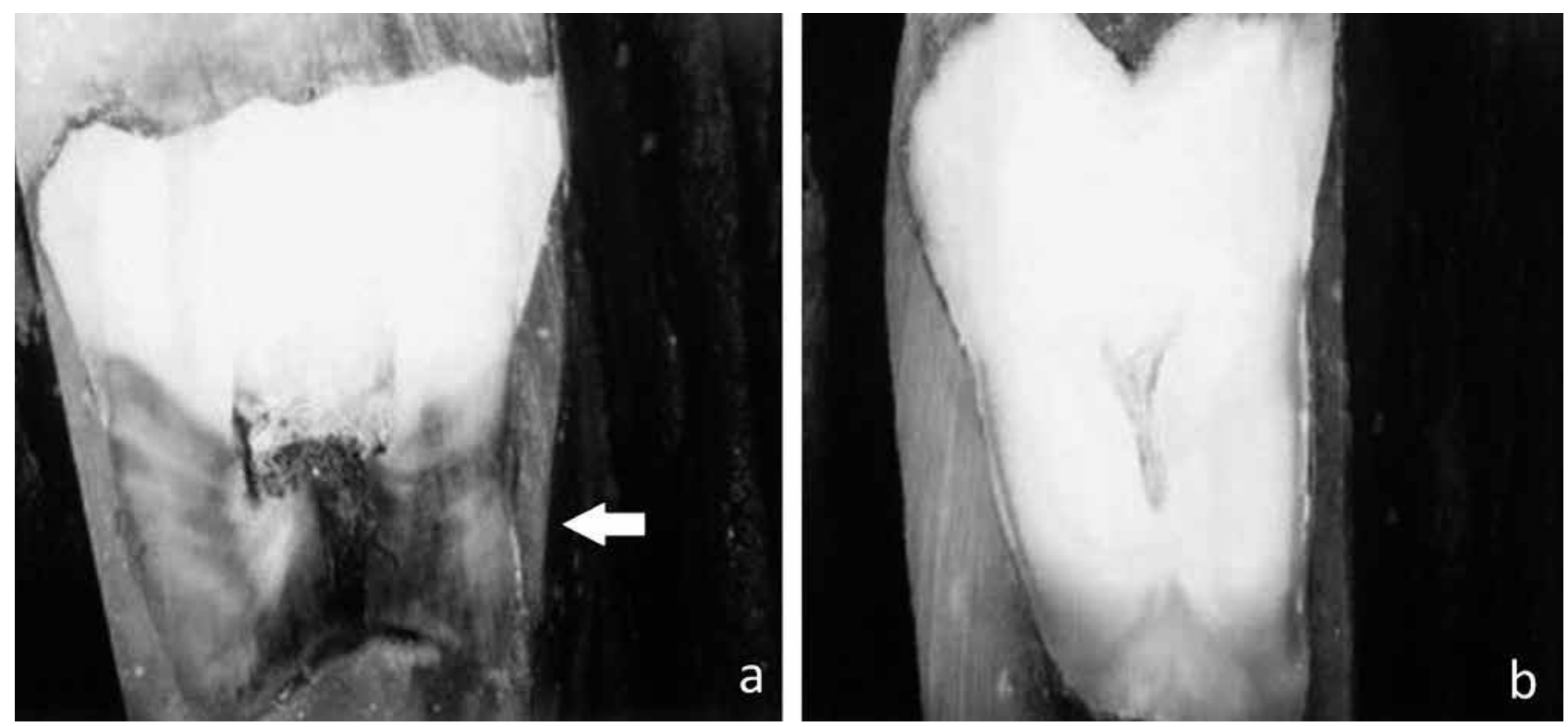

Fig. 1: (a) Positive control tooth showing complete penetration of dye solution throughout the perforation. Arrow shows dye penetration from the pulp chamber floor to the root apex. (b) Negative control tooth showing no penetration of the dye solution through the perforation. 
Tab. 1: Experimental results showing comparison of means and measures of central tendency for three groups

\begin{tabular}{|c|c|c|c|c|c|c|c|}
\hline & & Mean $\pm \mathrm{SD}^{*}$ & Median & Mode & Minimum & Maximum & $P$ value \\
\hline \multirow{3}{*}{$\begin{array}{l}\text { Dentin-cementum } \\
\text { thickness (mm) }\end{array}$} & Group A & $3.34 \pm 0.43$ & 3.35 & 2.60 & 2.60 & 3.90 & \multirow{3}{*}{$0.004 * *$} \\
\hline & Group B & $3.11 \pm 0.30$ & 3.15 & 2.80 & 2.60 & 3.60 & \\
\hline & Group C & $3.21 \pm 0.27$ & 3.30 & 3.40 & 2.80 & 3.60 & \\
\hline \multirow{3}{*}{$\begin{array}{l}\text { Micro-leakage } \\
(\mathrm{mm})\end{array}$} & Group A & $1.14 \pm 1.25$ & 0.85 & 0.20 & 0.20 & 3.70 & \multirow{3}{*}{0.332} \\
\hline & Group B & $1.66 \pm 0.86$ & 1.20 & 1.10 & 1.00 & 3.20 & \\
\hline & Group C & $2.83 \pm 0.62$ & 2.85 & 2.60 & 1.80 & 3.60 & \\
\hline \multirow{3}{*}{$\begin{array}{l}\text { Percentage } \\
\text { of leakage }(\%)\end{array}$} & Group A & $35.54 \pm 35.76$ & 25.40 & 100.00 & 5.26 & 100.00 & \multirow{3}{*}{$0.003 * *$} \\
\hline & Group B & $53.60 \pm 27.43$ & 37.83 & 100.00 & 29.17 & 100.00 & \\
\hline & Group C & $87.55 \pm 14.98$ & 95.16 & 100.00 & 62.07 & 100.00 & \\
\hline
\end{tabular}

* SD: Standard deviation $( \pm)$; ** Kruskal-Wallis test showing significant difference $(\mathrm{p}<0.05)$

\# Group A (Mineral trioxide aggregate); Group B (Calcium phosphate cement); Group C (Glass ionomer cement)

apical end to the pulp chamber floor of perforation was measured thrice from the micrometric scale on the ocular stage of the stereomicroscope. The average numerical value was taken as the final. For each section, the length of the perforation wall (dentin-cementum thickness) and the linear leakage of dye penetration were measured in millimeters $(\mathrm{mm})$. The ratio of linear leakage of dye penetration to the perforation wall length was expressed as a percentage for each selected section. Based on the percentage of depth of dye penetration, they were grouped as those that had penetrated $\geq 50 \%, 49-25 \%$ and $<25 \%$ of the total length.

All the specimens in each group were recorded in the similar manner, and the values were tabulated. All data were entered and analyzed using Statistical Package for Social Services, version 17.0 (SPSS Inc, IBM, IL, USA). Descriptive statistics and measures of central tendency were recorded. Kruskal-Wallis test and Chi-square test were used appropriately. The level of significance was set at $\mathrm{P}$ value $\leq 0.05$.

\section{Results}

The positive control Group-D exhibited complete dye penetration throughout the perforation while the negative control Group-E did not reveal any evidence of dye penetration (Fig. 1a,b). As the controls exhibited ideal staining, the accuracy of this test was established.

The dentin-cementum thickness varied significantly between the groups $(\mathrm{P}=0.004)$. In terms of linear length of the micro-leakage, Group $\mathrm{C}$ exhibited the highest leakage with $2.83 \mathrm{~mm}$ while Group A and B showed 1.14 and $1.66 \mathrm{~mm}$ respectively. The linear length of micro-leakage among the experimental groups revealed no significant difference $(P=0.332)$. It was observed that the mean leakage was $35.5 \%$ in the Group A, 53.6\% in Group B and the highest, $87.5 \%$ in Group C. The mean leakage percentage was statistically significant by Kruskal-Wallis test $(\mathrm{P}=0.003)$ when calculating the percentage of depth of leakage to the total length of perforation. The measures of central tendency and their comparison between the three experimental groups are shown in Table 1.

It was observed that comparing each depth of penetration of the dye, $50 \%$ of the samples in the Group A had less than $25 \%$ penetration while $40 \%$ of Group B cases had more than $50 \%$ dye penetration. In our study, all Group $\mathrm{C}$ teeth had $\geq 50 \%$ dye penetration. The difference between the groups was statistically significant $(\mathrm{P}=0.000)$ as shown in Table 2 .

Group A showed least degree of dye leakage as compared to the other two experimental groups, and the mean dye leakage was $1.14 \mathrm{~mm}$. The percentage of leakage was $35.5 \%$ as the dye leakage of 8 specimens out of 10 samples were confined within the perforation (Fig. 2A). Group B perforations showed relatively greater degree of dye penetration $(53.6 \%)$ compared to group A, and revealed that the dye leakage of 8 out of 10 specimens was confined within the perforation. The dye leakage had a mean length of $1.66 \mathrm{~mm}$ (Fig. 2B). Group C perforations showed maximum level of dye leakage at a mean length of $2.83 \mathrm{~mm}$ and the percentage of leakage was $87.5 \%$. The leakage of 5 specimens out of 10 samples was confined within the perforation (Fig. 2C).

Tab. 2: Comparison of depth of penetration between the three experimental groups

\begin{tabular}{|l|c|c|c|c|}
\hline $\begin{array}{l}\text { Depth of } \\
\text { penetration }\end{array}$ & $\begin{array}{c}\geq 50 \% \\
\mathrm{n}(\%)\end{array}$ & $\begin{array}{c}49-25 \% \\
\mathrm{n}(\%)\end{array}$ & $\begin{array}{c}<25 \% \\
\mathrm{n}(\%)\end{array}$ & \multirow{2}{*}{ P value } \\
\hline Group A & $2(20)$ & $3(30)$ & $5(50)$ & \multirow{2}{*}{$0.000 *$} \\
\hline Group B & $4(40)$ & $6(60)$ & - & \\
\hline Group C & $10(100)$ & - & - & \\
\hline
\end{tabular}

* Chi square test showing significant difference $(\mathrm{p}<0.05)$ \# Group A (Mineral trioxide aggregate); Group B (Calcium phosphate cement); Group C (Glass ionomer cement) 

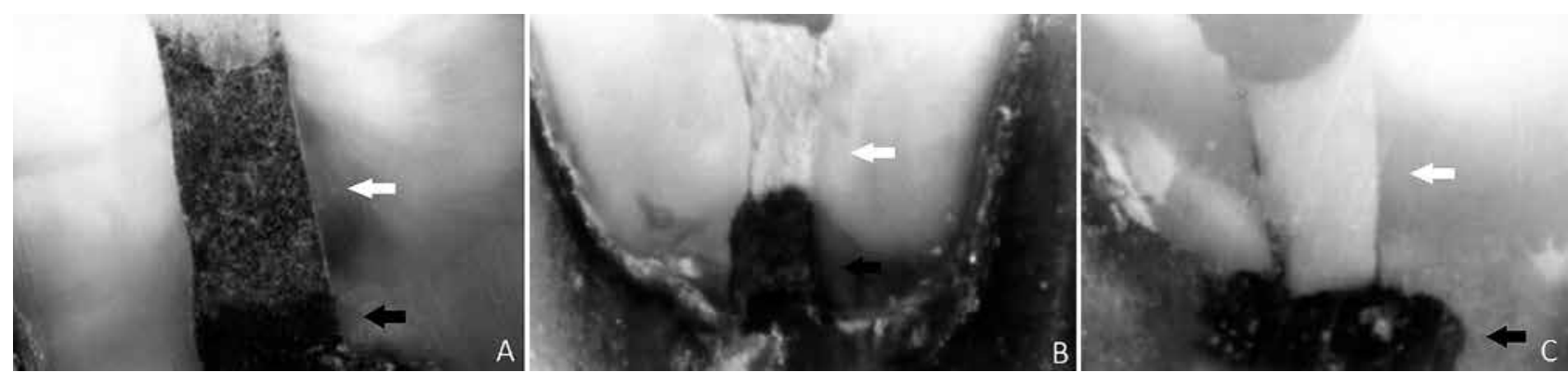

Fig. 2: (A) Perforation repaired with MTA (Group A). (B) Perforation repaired with CPC (Group B). (C) Perforation repaired with light cured GIC (Group C). White arrow shows the repair material placed in the perforation and black arrows points to the dye penetration.

\section{Discussion}

The principle goal of an endodontic therapy is to remove microbes and seal the root canal system effectively. Inadvertent perforation interferes with this goal because of damage to the periodontal attachment apparatus and subsequent bacterial proliferation. Perforations can be successfully managed with the use of a nonsurgical coronal approach by immediate placement of the reparative material in the perforation to prevent bacterial infection (18). The long-term success of a perforation repair is related to several factors among which, the foremost is the biocompatibility of the material and ability of repair material to provide an adequate seal (19).

The perforation of the pulpal floor into the furcation area of posterior teeth may occur iatrogenically due to inadequate knowledge of the pulp chamber anatomy, tooth malalignment or failure to consider anatomic variations. The calcification of the pulp chamber as a consequence of aging, trauma or other irritants may increase the risk of perforation during access and location of the canal orifices. Insufficient removal of the pulp chamber roof can also lead to perforation due to mishandling of the bur during access preparation. The non-iatrogenic causes of furcation perforation include internal resorption and dental caries (20). Furthermore, moisture, bleeding, unconventional access and a bottomless cavity makes repair of perforation a complicated procedure (14).

This study compares the sealing ability of MTA, self setting injectable CPC, and light cured GIC when used to repair furcation perforations. Many techniques including dyes (India ink, methylene blue), chemical tracers (silver nitrate, silver chloride), radioactive isotopes, neutron activation analysis, scanning electron microscopy and electrical conductivity have been recommended to test the sealing properties of restorative materials both in vivo and in vitro. The results of these studies have highlighted that margins of restorations are not fixed and inert with impenetrable borders and possess dynamic micro crevices which contain an active traffic of ions and molecules. The use of a dye is regarded as the easiest and most cost-effective method for detecting micro-leakage (21). Methylene blue dye was used in our study because it easily allows quantitative measurement of the area of dye penetration by linear measurement methods.

The results of the present study indicated that GIC had the greatest dye penetration $(87.55 \%)$ followed by CPC (53.6\%) and MTA (35.54\%). The sealing ability of MTA was therefore significantly better than the other two materials as evidenced by the high statistical significance $(\mathrm{P}=0.003)$. Still, even with the best MTA, one of every third such cases of perforation will have leakage that could lead to failure of the treatment. Dye leakage was confined within the perforation repair site in all except two specimens which showed total dye penetration. The presence of voids at the apical end and walls of the perforation may have caused total dye penetration in these two specimens which was later confirmed by stereomicroscopic examination.

The improved sealing ability of MTA is probably due to its hydrophilic nature and minimal setting expansion (22). Extrusion of MTA external to the furcation area was detected in 7 of 10 specimens. This extrusion of MTA into the defect would not cause any adverse effects as it has the ability to promote healing of periodontal tissues due to its bioactive nature when in direct contact with human tissues (10). An animal study conducted by Samiee et al. (23) proved that MTA induced mild inflammatory reactions and prompted hard tissue bridge formation when immediately used for furcal perforation repair.

Extrusion of CPC was observed in 8 of 10 specimens, and in some cases, the extrusion was rather pronounced. However, extruded CPC reportedly have no adverse affects on the healing of periodontal tissue in the area apical to the furcation defect (24). In fact, CPC may be effective in stimulating bone repair through osteoconduction and absorption as this aqueous based self-setting cement finally sets into hydroxyapatite, the mineral part of vertebrate bone. They possess the combined properties of osteoconductivity and biocompatibility of hydroxyapatite implants and the mouldability of acrylic bone cements and are highly versatile in 
application (25). A safe CPC cement formulation has been developed, and this new formulation is an injectable form with better viscosity and cohesive properties by incorporation of gelling agents. The cohesive property of CPC renders it suitable to seal a bleeding perforation site (26). In addition, high surface tension of the mixed cement allows only limited extrusion through the perforation (27).

A higher percentage of dye penetration in GIC may be due to the polymerization contraction of the material (28). Within the defect site, the cement was well adapted to one wall, but gaps were found on the opposite cavity wall. The site and type of dentin are also important factors as the dentin bond strength varies depending on the regional differences between wetness and chemical composition of the substrate (29). Contamination of the dentinal surface with excessive moisture, solvent or presence of voids could affect bonding, making it unpredictable under clinical conditions. There was extrusion of light cured GIC in most of the samples studied. This extrusion could produce detrimental effects in the prognosis of the teeth (30).

The mean leakage percentage of CPC and GIC was found to be lower in the present study compared to the results of a previous study (20). Furthermore, the authors concluded that CPC and GIC had equal sealing ability, whereas our results indicate that CPC had better sealing ability compared to GIC. The results of our study with regard to the number of specimens with signs of dye leakage may be compared to the in vitro study done by Daoudi and Sanders in which only $20 \%$ of the MTA specimens demonstrated dye leakage compared to our study of approximately $36 \%$. Similarly, the other experimental group was resin modified GIC which demonstrated signs of dye leakage in $90 \%$ of the specimens. This may be compared with our study in which approximately $87 \%$ specimens exhibited dye leakage with light cured GIC (31). A recent study also demonstrated better sealing ability of MTA when compared to biodentin and $\mathrm{CPC}$ by using the dye extraction leakage method (32).

A greater effort should be made, both in teaching and practice, regarding the avoidance of perforation thereby reducing damage to the periodontal ligament apparatus and preventing the dilemma of having a tooth with poor prognosis following injury.

\section{Conclusions}

From the results of the present study, it can be concluded that:

1. MTA showed the best sealing ability at perforation defects followed by CPC and GIC.

2. Extrusion of all three materials was observed in the majority of specimens indicating that adequate care should be taken in this regard.

3. Voids along the walls and at the apical region were also responsible for failure of sealing ability of the restoration.
The mineral trioxide aggregate and calcium phosphate cement with its added properties like biocompatibility and osteoconduction may significantly improve the prognosis of teeth with furcation perforation defect. The direct extrapolation of the results to the clinical situation needs to be undertaken with caution only after further in vivo investigations.

\section{References}

1. Mittal M, Chandra S. An evaluation of plaster of Paris barriers used under various materials to repair furcation perforations (in vitro study). J Endod 1999; 25: $385-8$.

2. Ingle JI. A standardized endodontic technique utilizing newly designed instruments and filling materials. Oral Surg Oral Med Oral Pathol 1961; 14: 83-91.

3. Lantz B, Persson PA. Periodontal tissue reactions after surgical treatment of root perforations in dogs' teeth. A histologic study. Odontol Revy 1970; 21: 51-62.

4. Tsesis I, Fuss Z. Diagnosis and treatment of accidental root perforations. Endod Topics 2006; 13: 95-107.

5. Oswald RJ. Procedural accidents and their repair. Dent Clin North Am 1979; 23 : 593-616.

6. Rafter M, Baker M, Alves M, Daniel J, Remeikis N. Evaluation of healing with use of an internal matrix to repair furcation perforations. Int Endod J 2002; 35: 775-83.

7. Alhadainy HA. Root perforations. A review of literature. Oral Surg Oral Med Oral Pathol 1994; 78: 368-74.

8. Lee SJ, Monsef M, Torabinejad M. Sealing ability of a mineral trioxide aggregate for repair of lateral root perforations. J Endod 1993; 19: 541-4.

9. Pitt Ford TR, Torabinejad M, McKendry DJ, Hong C-U, Kariyawasam SP. Use of mineral trioxide aggregate for repair of furcal perforations. Oral Surg Oral Med Oral Pathol Oral Radiol Endod 1995; 79: 756-63.

10. Parirokh M, Torabinejad M. Mineral trioxide aggregate: a comprehensive literature review - Part III: Clinical applications, drawbacks, and mechanism of action. J Endod 2010; 36: 400-13.

11. Ambard AJ, Mueninghoff L. Calcium phosphate cement: review of mechanical and biological properties. J Prosthod 2006; 15: 321-8.

12. Noetzel J, Ozer K, Reisshauer B-H, et al. Tissue responses to an experimental calcium phosphate cement and mineral trioxide aggregate as materials for furcation perforation repair: a histological study in dogs. Clin Oral Invest 2006; 10: $77-83$.

13. Anstice H, Nicholson J. Studies on the structure of light-cured glass-ionomer cements. J Mater Sci 1992; 3: 447-51.

14. Alhadainy HA, Himel VT. Evaluation of the sealing ability of amalgam, Cavit, and glass ionomer cement in the repair of furcation perforations. Oral Surg Oral Med Oral Pathol 1993; 75: 362-6.

15. Alhadainy HA, Himel VT. Comparative study of the sealing ability of light-cured versus chemically cured materials placed into furcation perforations. Oral Surg Oral Med Oral Pathol 1993; 76: 338-42.

16. Kumar M, Sequeira PS, Peter S, Bhat GK. Sterilisation of extracted human teeth for educational use. Indian J Med Microbiol 2005; 23: 256-8.

17. Kohli A, Puttaiah R, Bedi R, et al. Infection Control \& Occupational Safety Recommendations for Oral Health Professionals in India. 1st ed. New Delhi: Dental Council of India; 2007.

18. Friedman S. Considerations and concepts of case selection in the management of post-treatment endodontic disease (treatment failure). Endod Topics 2002; 1: $54-78$.

19. Holland R, Filho JA, de Souza V, Nery MJ, Bernabe PF, Junior ED. Mineral trioxide aggregate repair of lateral root perforations. J Endod 2001; 27: $281-4$.

20. Chau JY, Hutter JW, Mork TO, Nicoll BK. An in vitro study of furcation perforation repair using calcium phosphate cement. J Endod 1997; 23: 588-92.

21. Lolayekar N, Bhat SS, Hegde S. Sealing ability of ProRoot MTA and MTA-Angelus simulating a one-step apical barrier technique-an in vitro study. J Clin Pediatr Dent 2009; 33: 305-10.

22. Torabinejad M, Chivian N. Clinical applications of mineral trioxide aggregate. J Endod 1999; 25: 197-205.

23. Samiee M, Eghbal MJ, Parirokh M, Abbas FM, Asgary S. Repair of furcal perforation using a new endodontic cement. Clin Oral Invest 2010; 14: 653-8.

24. Telli C, Serper A, Dogan AL, Guc D. Evaluation of the cytotoxicity of calcium phosphate root canal sealers by MTT assay. J Endod 1999; 25: 811-3.

25. Fujikawa K, Sugawara A, Murai S, Nishiyama M, Takagi S, Chow L. Histopathological reaction of calcium phosphate cement in periodontal bone defect. Dent Mater J 1995; 14: 45-57.

26. Komath M, Varma H. Development of a fully injectable calcium phosphate cement for orthopedic and dental applications. Bull Mater Sci 2003; 26: 415-22. 
27. Ishikawa K, Asaoka K. Estimation of ideal mechanical strength and critical porosity of calcium phosphate cement. J Biomed Mater Res 2004; 29: 1537-43.

28. Chong BS, Pitt Ford TR, Watson TF. The adaptation and sealing ability of lightcured glass ionomer retrograde root fillings. Int Endod J 1991; 24: 223-32.

29. Himel VT, Alhadainy HA. Effect of dentin preparation and acid etching on the sealing ability of glass ionomer and composite resin when used to repair furcation perforations over plaster of Paris barriers. J Endod 1995; 21: 142-5.

30. Alhadainy HA, Himel VT. An in vitro evaluation of plaster of Paris barriers used under amalgam and glass ionomer to repair furcation perforations. J Endod 1994; 20: 449-52.

31. Daoudi MF, Saunders WP. In vitro evaluation of furcal perforation repair using mineral trioxide aggregate or resin modified glass ionomer cement with and without the use of the operating microscope. J Endod 2001; 28: 512-15.

32. Sanghavi T, Shah N, Shah RR. Comparative analysis of sealing ability of biodentin and calcium phosphate cement against mineral trioxide aggregate (Mta) as a furcal perforation repair material (an in vitro study). NJIRM 2013; 4: 56-9.

Received: $21 / 04 / 2013$

Corresponding author:

Accepted in revised form: 11/09/2013

Dr Nimmi Abraham, Dental Caries Research Chair, College of Dentistry, King Saud University, P.O. Box 60169, Riyadh 11545, Saudi Arabia; e-mail: nimmibaksu@gmail.com 